\title{
Effects of number of CS-US pairings on the strength of conditioned frustration in rats
}

\author{
PETER C. SENKOWSKI and VALERIE A. VOGEL \\ Northern Illinois University, DeKalb, Illinois 60115
}

\begin{abstract}
Following 45 placements to a wet-mash reward, groups of rats received $0,10,20$, or 40 frustration-conditioning trials during which primary frustration was paired with the apparatus cues plus a distinctive CS. Other groups received 0,10 , or 20 pairings of primary frustration and apparatus cues alone. In the CS condition, learning of a hurdle-jumping response which terminated the CS was nonmonotonically related to the number of frustration-conditioning trials on the first of 2 test days. Performance increased with the number of conditioning trials up to 20, then decreased following 40 such trials. Among groups which did not receive the $\mathrm{CS}$ during conditioning or testing, only subjects in the zero-pairing condition showed evidence of learning. The results were interpreted as supporting the conclusion that conditioned frustration had acquired aversive motivational properties since its effectiveness was found to vary systematically with the number of conditioning trials. It was further concluded that the superiority of hurdle-jumping performance by CS groups relative to NCS groups was the result of both a higher level of frustrative motivation and greater frustration reduction following the response for the former groups.
\end{abstract}

Amsel's (1958) frustration theory states that the occurrence of nonreward in the presence of stimuli previously associated with reward results in an aversive emotional response of unconditioned, or primary, frustration $\left(R_{F}\right)$. It is also held that the classical pairing of a neutral stimulus (CS) and $R_{F}$ will result in that stimulus's gaining the capacity to elicit frustration in its conditioned form ( $\left.r_{F}\right)$. Since this anticipatory form of frustration is assumed to have the capacity to energize behavior occurring in its presence and to reinforce behavior through its reduction, $\mathrm{r}_{\mathrm{F}}$ may be given the status of an acquired or learned motive. Moreover, as a learned motive, the strength of $\mathrm{r}_{\mathrm{F}}$ should vary as a function of commonly manipulated learning variables such as number of reinforced conditioning trials, interstimulus interval during conditioning, etc. (cf. Brown, 1961, chap. 5).

Wagner (1963) reported one of the first experiments designed to examine the energizing and reinforcing properties of $r_{F}$. Rats in Wagner's experiment performed a hurdle-jumping response which resulted in escape from a stimulus previously paired with frustrative nonreward. However, there was no increase in their hurdle-jumping speeds over trials, and differences in performance between the experimental group and a control group which had not had the CS paired with $R_{F}$ was due to a decrease in speed over trials by the latter group. Therefore, while

Thanks are extended to Dorothy McAllister and to Wallace McAllister for a critical reading of the manuscript. Requests for reprints should be sent to Peter C. Senkowski, Department of Psychology, Northern Illinois University, DeKalb, Illinois 60115 .
Wagner's study demonstrated that a CS paired with $R_{F}$ could serve to energize behavior, the assumption that reduction of $r_{F}$ would serve as a reinforcer for the hurdle-jumping response was not unequivocally supported.

Wagner's hurdle-jumping technique was modified by Daly (e.g., Daly, 1969, 1970) and was shown to be useful in testing a number of predictions derived from frustration theory. In one of the paradigms employed by Daly, hungry rats are first given a number of consistently rewarded placements in the startbox of a hurdle-jumping apparatus so that an expectancy of reward $\left(r_{R}\right)$ can be conditioned to the start-box stimuli. These rewarded placements are followed by a number of nonrewarded placements in the presence of a distinctive CS. The purpose of the nonrewarded placements is to provide for the classical pairing of the $C S$ and $R_{F}$ so that the $C S$ will gain the capacity to elicit $r_{F}$. Finally, subjects are placed in the unbaited startbox with the CS present and allowed to escape the CS by jumping a hurdle into an adjacent safe box. The learning of the hurdle-jumping response, as indexed by increasing response speeds over trials, is interpreted as being motivated by frustration and reinforced by frustration reduction.

In Daly's procedure, subjects receive both frustration conditioning and testing in the same apparatus. Therefore, the motivator for the hurdle-jumping response could include not only frustration conditioned to the CS, but also $R_{F}$ as well as any $r_{F}$ which had become associated with apparatus cues. In order to isolate and assess the motivational effects of $\mathrm{r}_{\mathrm{F}}$ conditioned to the discrete CS, Daly (1969) included a group which was never exposed to the CS during 
either nonrewarded placements or testing (NCS condition). While both groups learned the hurdle-jumping response, the performance of NCS subjects was inferior to that of CS subjects. It was concluded that the conditioning trials were effective in making the $\mathrm{CS}$ an elicitor of frustration.

Daly's (1969) findings are certainly consistent with the assumption that $r_{F}$ is a learned motive. At the present time, however, there is little systematic data which indicate either the relevant parameters or the limits of the conditioning process. The present experiment was designed to assess the relationship between $r_{F}$, as indexed by speed of hurdle jumping, and the number of CS- $R_{F}$ pairings preceding testing. The initial design of the experiment included six groups of subjects which differed in both the number of nonrewarded placements which preceded testing $(0,10$, or 20$)$, and in whether or not a CS was present during those nonrewarded placements and subsequent testing (CS vs. NCS). The nonrewarded placements will hereafter be referred to as frustrationconditioning trials. An additional group of subjects receiving 40 frustration-conditioning trials with the CS prior to testing was run subsequently to extend the range of conditioning trials.

\section{METHOD}

\section{Subjects}

Ninety naive, male hooded rats, 100-120 days cld at the start of the experiment, were obtained from a colony maintained by the Psychology Department. Subjects were individually housed, and following a 2-week period to allow body weights to stabilize, reduced to $80 \%$ of their ad-lib weight through restrictive feeding. This level of deprivation was maintained throughout the experiment. Water was continuously available, except when subjects were in the experimental apparatus. A randomized block procedure based on ad-lib body weights was used to assign subjects to one of the initial six groups $(n=15)$. After data collection had been completed, an additional group of 15 subjects from the same source was assigned to a CS condition in which $\mathbf{4 0}$ frustration-conditioning trials were administered prior to testing.

\section{Apparatus}

A one-way hurdle-jumping apparatus was used in all phases of the experiment. It consisted of a startbox and a safe box, each of which was $29.2 \mathrm{~cm}$ long, $11.1 \mathrm{~cm}$ wide, and $14.0 \mathrm{~cm}$ high. The two boxes were separated by a vertically sliding door $(10.0 \times 6.0 \mathrm{~cm})$ resting on a hurdle which was $3.5 \mathrm{~cm}$ high. The startbox was painted flat white with a solid wooden floor. The walls were lined with clear Plexiglas. A bent teaspoon, which hung on a wall of the startbox $2.5 \mathrm{~cm}$ above the floor and $5.0 \mathrm{~cm}$ from the hurdle door, served as the food cup. A photobeam was aimed across the startbox, $3.5 \mathrm{~cm}$ above the floor and $10 \mathrm{~cm}$ from the hurdle door. Breaking this photobeam started a clock which was used to measure the time subjects had access to the baited food cup during rewarded placements. The safe box was painted dark gray, and had a solid wooden floor covered by hardware cloth. A photobeam was aimed across the safe box, $3.5 \mathrm{~cm}$ above the floor and $10 \mathrm{~cm}$ from the hurdle. A Hunter Klockounter measured hurdle-jumping latencies in $.01 \mathrm{sec}$ from the opening of the door until the photobeam in the safe box was broken. Two boxes, with dimensions identical to those of the start and goal boxes, served as covers for those compartments. Each of these cover boxes had a Plexiglas bottom, above which was mounted a 7.5-W lamp to provide general illumination. The cover of the startbox also housed a $40-W$ lamp which served as the CS. The Plexiglas bottom of the startbox cover was sanded, while that of the safebox was clear and covered by hardware cloth.

\section{Procedure}

Subjects were run in squads of three, with all the subjects in a squad being assigned to the same group. On the first day of the experiment, each subject was handled for $2 \mathrm{~min}$, after which it was allowed to explore the apparatus for $5 \mathrm{~min}$ with the hurdle door open. Following handling and exploration, the subjects received $2 \mathrm{~g}$ of wet mash in their home cages prior to receiving the balance of their daily food ration 20 min later. On each of the next 3 days, subjects received 15 rewarded placements in the startbox. Each placement consisted of 20-sec access to wet mash. The food cup was filled to capacity prior to each rewarded placement, and was never observed to be less than half filled at the end of a placement. Subjects of a squad were run in rotation, and an intertrial interval of $2 \mathrm{~min}$ was maintained throughout the experiment. Following the last placement of each day, subjects were returned to their home cages where they received the balance of their daily food ration $20 \mathrm{~min}$ later. On the following day, subjects received 0,10 , or 20 frustration-conditioning trials in the startbox with an empty food cup. Subjects in group CS -40 received 20 conditioning trials on each of 2 successive days. Each frustration-conditioning trial was $10 \mathrm{sec}$ long. For subjects in the CS groups, the $40-W$ lamp came on with the placement of the subject in the apparatus, and terminated when the subject was removed.

Hurdle-jumping testing for groups receiving zero frustrationconditioning trials began the day immediately following the last rewarded placement, whereas testing for the other groups began the day following the last frustration-conditioning trial. On each of the 2 testing days, subjects received 15 hurdle-jumping trials. On each trial, the subject was placed in the startbox with the empty food cup. After the subject had oriented toward the door, or after $10 \mathrm{sec}$ had elapsed since being placed in the apparatus, the hurdle door was raised and the Klockounter started. For subjects in CS groups, opening of the hurdle door was accompanied by onset of the CS. A hurdle-jumping response, defined by the breaking of the photobeam in the safe box, resulted in the stopping of the Klockounter and termination of the CS. The hurdle door was lowered to prevent retracing, and the subject was allowed to remain in the safe box for $10 \mathrm{sec}$ before being returned to the holding cage. If no response occurred within $\mathbf{4 0 ~} \mathrm{sec}$ of the raising of the door, the subject was returned to the holding cage and a latency of $40 \mathrm{sec}$ was recorded.

\section{RESULTS}

Hurdle-jumping latencies were converted to speeds by reciprocal transformation and were averaged over blocks of three trials. Response speeds for NCS groups are presented in the top half of Figure 1, and those for CS groups, in the bottom half for Day 1 and Day 2 of hurdle-jumping testing. The initial analyses did not include the data from group CS-40. The performance of that group relative to that of the other CS groups will be examined subsequently.

Day 1 data show that the speeds of the CS and NCS groups diverged over blocks of testing trials, with CS groups showing a general increase in response speeds and NCS groups exhibiting successive declines in performance. An analysis of variance of these data used the number of frustration-conditioning trials 


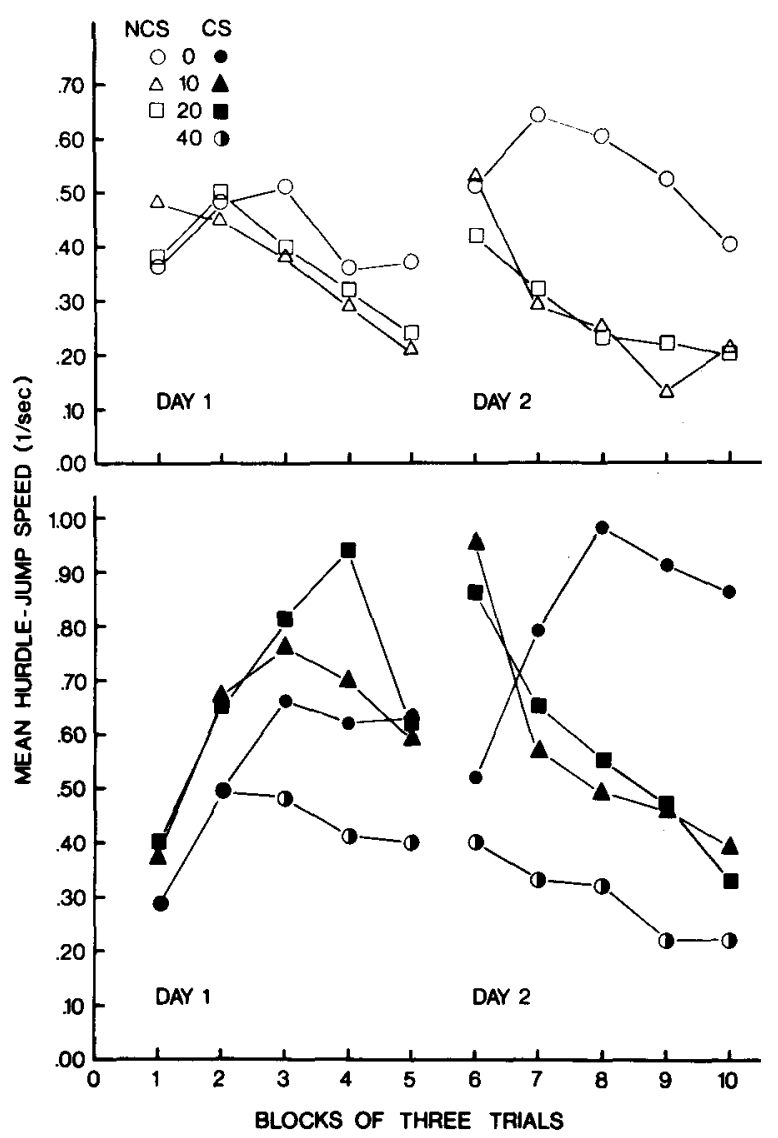

Figure 1. Mean speed of hurdle jumping per three trial blocks for Day 1 and Day 2 of hurdle-jumping testing (15 trials/day).

received prior to testing $(\mathrm{N})$ and presence or absence of the CS (CS-NCS) as between-subjects factors and blocks of test trials (Blocks) as the within-subjects factor. Consistent with the visual impression given in Figure 1, a reliable CS-NCS by Blocks interaction was obtained, $F(4,336)=14.33, p<.001$. Simple effects tests showed no reliable differences in response speeds between CS and NCS groups on the first block of trials, $F(1,84)=2.00, p>.10$, but indicated that CS groups performed at a higher level than did NCS groups on Blocks 2 through 5, $F s(1,84)=4.00,7.33,34.75$, and 32.25 , respectively. These differences were reliable at beyond the .05 level of significance on Block 2, and at beyond the .001 level on the remaining three blocks of trials. The effect of the number of frustration-conditioning trials was not statistically reliable, nor did this factor interact with either of the other two variables. Treatment by Subjects analyses of variance for each group indicated reliable effects of trial blocks for all three CS groups (ps $<.005$ ). Although the analyses of data from groups NCS-10 and NCS-20 also indicated reliable trial blocks effects ( $p s<.025$ ), there was no reliable variation in speeds over blocks of trials for group NCS-0. The results of these analyses, in conjunction with inspection of Figure 1, support the conclusion that all CS groups showed an increase in hurdle-jumping speeds over the first test day while groups NCS-10 and NCS-20 exhibited general decreases in speed over trial blocks.

The data obtained from Day 2 of hurdle jumping were subjected to an analysis of variance with CS-NCS and $\mathrm{N}$ as between-subjects factors and Blocks as the within-subjects factor. The three-way interaction was found to be statistically reliable, $F(8,336)=2.22$, $\mathrm{p}<.025$, and was further investigated by performing separate CS-NCS by $\mathrm{N}$ analyses of variance for each trial block. The results of these analyses indicated that CS groups jumped reliably faster than did NCS groups on every trial block (ps $<.005$ ). The effect of number of frustration-conditioning trials was significant on Blocks 7 through 10 . Further analyses revealed that on Block 7 , groups receiving zero frustration-conditioning trials jumped faster than those receiving 10 conditioning trials $(p<.05)$, and marginally faster than groups receiving 20 such trials (p $<.10)$. On Blocks 8 through 10 , the zero-trial groups jumped faster than groups receiving 10 or 20 conditioning trials $(p s<.01)$. The speeds of the groups receiving 10 or 20 conditioning trials were never found to differ reliably from one another (ps > .50).

Additional analyses compared the performance of CS and NCS groups receiving the same number of conditioning trials over trial blocks on Day 2. For groups receiving either 10 or 20 frustrationconditioning trials prior to testing, CS subjects jumped faster than did NCS subjects, Fs $(1,28)=21.47$ and 33.00 , ps $<.001$, for the 10 - and 20 -conditioningtrial groups, respectively. The decline in performance over trial blocks was also reliable in both these analyses (ps $<.001$ ). A significant CS-NCS by Blocks interaction, $F(4,112)=2.84, p<.05$, was found in the analysis of data from groups receiving zero conditioning trials prior to testing. Subsequent Treatment by Subjects analyses of variance found a reliable increase in response speed over trial blocks for group CS-0 $(p<.001)$, but no variation in performance over blocks of trials for group NCS-0 $(p>.10)$.

Although separate analyses of data from the 2 days of hurdle-jumping testing failed to detect changes in speeds over trial blocks for subjects in group NCS-0, inspection of Figure 1 suggests that some learning had occurred in this group. To examine this possibility, a Treatment by Subjects analysis of variance was performed using the data from all 10 trial blocks for this group. The effect of trial blocks was found to be statistically reliable, $F(9,126)=2.34$, $p<.025$. Comparisons of performance on each trial block with that on Block 1 found response speeds on Blocks 7 and 8 to be faster than on Block 1 
(ps $<.005$ ), supporting the conclusion that some learning had occurred in this group.

Data obtained from the CS groups were reanalyzed so as to include those of group CS-40. An analysis of variance of hurdle-jumping speeds from Day 1 found reliable effects of both $N, F(3,56)=4.81$, $p<.01$, and Blocks, $F(4,224)=19.17, p<.001$. Since a nonmonotonic relationship between the number of frustration-conditioning trials and hurdle-jumping performance could be expected on the basis of arguments to be presented later, trend tests were conducted using the grand means of hurdle-jumping performance on Day 1 of testing. Those means are presented in Figure 2. These analyses found only the quadratic trend component to be statistically reliable, $F(1,56)=11.14, p<.005$. Of the variance attributable to differences between groups, $77 \%$ could be accounted for by the quadratic trend.

An analysis of variance of hurdle-jumping speeds for all of the CS groups on Day 2 of testing found a reliable $\mathrm{N}$ by Blocks interaction, $\mathrm{F}(12,224)=6.00$, $\mathrm{p}<.001$. One-way analyses of variance comparing the groups on each trial block revealed a reliable effect of number of frustration-conditioning trials on every block ( $p s<.01)$. Subsequent comparisons used the Newman-Keuls procedure (Winer, 1962, p. 309). The following differences between groups were reliable at beyond the .05 level of significance. On Block 6, groups CS-10 and CS-20 both jumped reliably faster than did group CS-40, but did not differ from one another. Group CS-10 was also

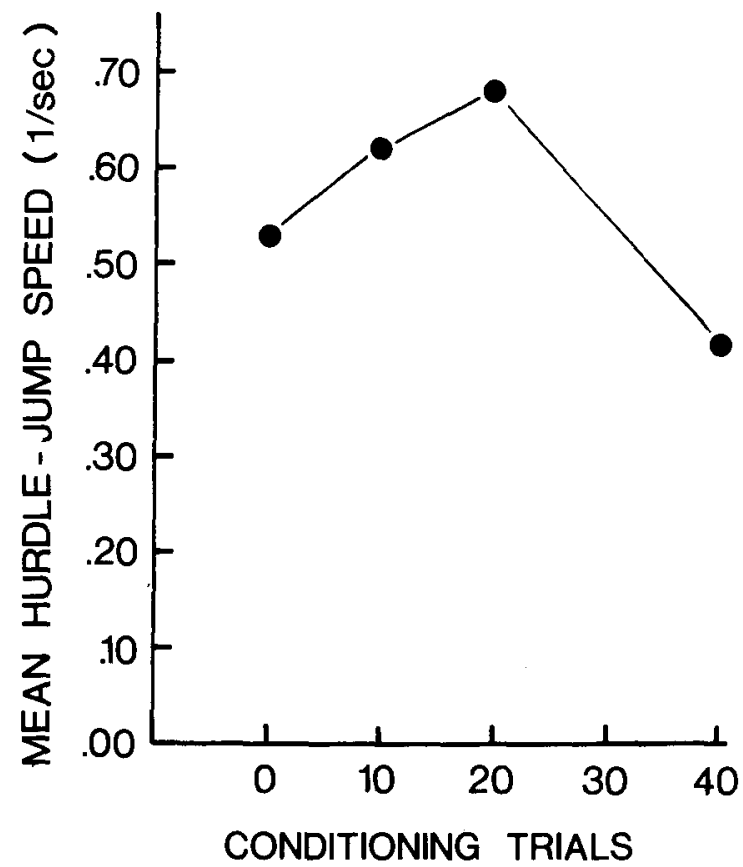

Figure 2. Grand means of hurdle-jumping speeds for each $\mathrm{CS}$ group on Day 1 of hurdle-jumping testing. found to jump faster than group CS-0. On Block 7, group CS-40 was slower than any of the other groups, which were not found to differ from one another. Group CS-0 performed at a higher level than any of the other groups on Blocks 8, 9, and 10. In addition, groups CS-10 and CS-20 both jumped faster than group CS-40 on Block 9.

\section{DISCUSSION}

One assumption of frustration theory (Amsel, 1958 ) is that a CS paired with primary frustration can, through a conditioning process, acquire the capacity to motivate behavior. The present experiment attempted to test directly the assumption that $r_{F}$ is a learned motive by varying the number of classical $C S-R_{F}$ pairings administered prior to hurdle-jumping testing. In any attempt to directly examine the conditioning of $r_{F}$, it must be recognized that the procedure involves a step not required in the conditioning of other responses. Prior to the conditioning of $r_{F}$, it is necessary to condition $r_{R}$ to the apparatus cues so that nonreward will in fact elicit $R_{F}$. It is only after $r_{R}$ has sufficiently developed that the presentation of a CS in the presence of nonreward can be expected to result in the conditioning or $r_{F}$, A problem is that, while CS- $R_{F}$ pairings are taking place, $r_{R}$ is undergoing extinction because its US (food) is no longer present. Since the magnitude of $R_{F}$ is assumed to vary directly with the strength of $r_{R}$, each successive conditioning trial will result in the CS being paired with a relatively weaker $R_{F}$. If enough such pairings are administered, eventually the CS will be presented without the US of $R_{F}$ and hence will result in the extinction of $\mathrm{r}_{\mathrm{F}}$.

Given the above reasoning, the nonmonotonic relationship obtained between the number of frustration-conditioning trials and the performance of the CS groups on Day 1 is in full accord with predictions derived from frustration theory. The poor performance of group CS-0 relative to that of groups CS-10 and CS-20 on Day 1 can be attributed to one or both of two possible factors. First, since groups receiving zero-conditioning trials experienced $R_{F}$ for the first time during the testing trials of Day 1 , it might be expected that frustration-elicited behaviors (e.g., attacking the food cup, biting the door opening) would interfere with the performance of the hurdle-jumping response, thus resulting in low performance levels initially. In addition, subjects in group CS-0 had no opportunity to have $\mathrm{r}_{\mathrm{F}}$ conditioned to the CS prior to the start of testing. Until the test trials themselves could allow for the conditioning of $r_{F}$ to the CS, performance in group CS-0 would presumably be mediated exclusively by $R_{F}$. The data reveal that the performance of groups CS-0 and NCS-0 is quite similar on Day 1, a finding which 
would be predicted if the hurdle jumping of both groups were being motivated primarily by $R_{F}$. That the testing trials on Day 1 did serve to condition $r_{F}$ to the CS is suggested by the performance of group CS-0 on Day 2. While other CS groups showed a systematic decline in performance over blocks of trials, which presumably reflects the extinction of $r_{F}$, group CS-0 showed an increase in hurdlejumping speed.

Even though $r_{R}$ could be assumed to be extinguishing during the course of the frustration-conditioning trials, the relationship between the hurdle-jumping performance of the CS groups and the number of conditioning trials was direct over 0,10 , and 20 such trials. This finding lends strong support to the conclusion that increasing numbers of $C S-R_{F}$ pairings were effective in increasing the capacity of the CS to elicit $\mathrm{r}_{F}$ within this range. The performance of group $\mathrm{CS}-40$ is also consistent with a frustration interpretation. The extended number of nonrewarded placements received by subjects in this group prior to testing would presumably result in more complete extinction of $r_{R}$, thus eliminating $R_{F}$ and $r_{F}$ as motivators and reinforcers for the hurdle-jumping response.

As predicted by frustration theory, the hurdlejumping performance of CS groups was superior to that of comparable NCS groups throughout most of testing. This finding suggests that CS groups experienced a higher level of frustrative motivation than did comparable NCS groups. It is reasonable to assume that $\mathrm{r}_{\mathrm{F}}$ conditioned to the $\mathrm{CS}$ during the frustration-conditioning trials could of fset, at least partially, any loss of $R_{F}$ that would be expected to occur over the course of those trials. An additional factor favoring superior performance by the CS groups would be the amount of reinforcement, through frustration reduction, which followed a hurdle-jumping response. If amount of reinforcement can be equated with degree of stimulus change, as has been done with regard to fear conditioning (e.g., McAllister, McAllister, Brooks, \& Goldman, 1972), then the termination of the CS which followed a response in the $C S$ groups would constitute a greater amount of reinforcement than was available for subjects in the NCS conditions.

Another possible reason for performance differences between CS and NCS groups could be factors intrinsic to the CS. Potentially, the light used as a CS could have elicited unconditioned escape behaviors which could have been reinforced by CS termination. The failure of group CS-40 to show any learning of the hurdle-jumping response argues against this interpretation, as does the finding that CS and NCS groups did not differ on the first block of test trials. If the CS were intrinsically aversive, its effects should have been present early in testing, especially in group CS- 0 .
While it has been assumed that $r_{F}$ conditioned to the CS was the primary determinant of hurdlejumping performance in the CS groups, it is possible that any $R_{F}$ which remained after the frustrationconditioning trials had been administered, as well as any $r_{F}$ associated with apparatus cues, could also serve to motivate performance. An examination of the performance of the NCS groups should reveal the extent to which these potential sources of motivation were effective. Inspection of the top half of Figure 1 shows that groups NCS-10 and NCS-20 exhibited reliable declines in performance over trial blocks. This would seem to indicate that $R_{F}$ had dissipated appreciably by the time the last frustration-conditioning trial had been completed, and that any $r_{F}$ which had been conditioned to the apparatus cues was insufficient to mediate learning.

Of the NCS groups, only group NCS-0 showed any evidence of learning. It might be assumed that the testing trials on Day 1 served to condition $r_{F}$ to the apparatus cues and that it was this source of motivation which mediated the hurdle jumping of group NCS-0 on Day 2. If this were the sole determinant of hurdle jumping in this group, however, it might be predicted that the level of performance of group NCS-0 on Day 2 would be similar to that of groups NCS-10 and NCS-20 on Day 1. Inspection of the top half of Figure 1 shows that this was not the case. The performance of group NCS-0 on Day 2 is clearly superior to that of the other two NCS groups on Day 1. A reasonable interpretation of this result is in terms of differences in the amount of $r_{R}$ and hence of $R_{F}$ experienced by the three groups during testing trials. As can be seen in Figure 1, subjects in group NCS- 0 were performing the hurdle-jumping response on Day 1 with latencies considerably less than $10 \mathrm{sec}$, which was the duration of exposure on each frustration-conditioning trial for the NCS-10 and NCS-20 groups. This means that the total amount of exposure to nonreward in the context of startbox stimuli over the course of testing on Day 1 was less in group NCS-0 than it was during the frustrationconditioning trials of the other two NCS groups. Therefore, it would be reasonable to expect that the extinction of $r_{R}$ would be less by Day 2 for group NCS-0 than on Day 1 for the other two groups. Hence, the magnitude of $R_{F}$ available for mediating hurdle-jumping performance in group NCS-0 on Day 2 would be appreciably higher than that available to groups NCS-10 and NCS-20 on Day 1. Given this explanation, the poor performance of group NCS-0 on Day 1 would not be expected. However, as suggested earlier, experiencing $R_{F}$ for the first time on Day 1 may have resulted in the elicitation of frustration-related behaviors which interfered with performance of the hurdle-jumping response on that day. It would be expected that such competing responses would extinguish over the course of testing 
on Day 1, since they would result in delay of reinforcement. If the above interpretation is correct, it is reasonable to assume that $\mathbf{R}_{\mathbf{F}}$ would also have contributed to the performance level of group CS- 0 . However, the superiority of the CS-0 group over the NCS- 0 group can be attributed to the conditioning of frustration to the CS.

The performance of the NCS groups in the present experiment was similar in most respects to that of comparable groups in an experiment by Brooks and Goldman (1971). In that experiment, designed to examine the effects of continuous nonreward on $R_{F}$, a group comparable to group NCS-0 was found to show some evidence of learning. As in the present experiment, groups receiving either 10 or 20 nonrewarded placements without a CS prior to testing demonstrated no learning.

The results of the present experiment lend further support to the prediction of frustration theory that $R_{F}$ and $r_{F}$ can serve to motivate behavior, and in addition that termination of frustration can serve as a reinforcer. The manipulation of a fundamental learning variable, the number of CS-R $R_{F}$ pairings, resulted in findings which support the conclusion that $r_{F}$, like fear, can be given the status of a learned or acquired motive.

\section{REFERENCES}

AMSEL, A. The role of frustrative nonreward in noncontinuous reward situations. Psychological Bulletin, 1958, 55, 102-119.

Brooks, C. I., \& Goldman, J. A. Changes in the intensity of primary frustration during continuous nonreward. Journal of Experimental Psychology, 1971, 90, 153-155.

BRown, J. S. The motivation of behavior. New York: McGraw-Hill, 1961.

DALY, H. B. Learning of a hurdle-jump response to escape cues paired with reduced reward or frustrative nonreward. Journal of Experimental Psychology, 1969, 79, 146-157.

DALY, H. B. Combined effects of fear and frustration on acquisition of a hurdle-jump response. Journal of Experimental Psychology, $1970,83,89-93$.

McAllister, D. E., McAllister, W. R., Brooks, C. I., \& Goldman, J. A. Magnitude and shift of reward in instrumental aversive learning in rats. Joumal of Comparative and Physiolog. ical Psychology, 1972, 80, 490-501.

W AGNER, A. R. Conditioned frustration as a learned drive. Journal of Experimental Psychology, 1963, 66, 142-148.

WINER, B. J. Statistical principles in experimental design. New York: McGraw-Hill, 1962.

(Received for publication February 26, 1976; revision accepted July $16,1976$. ) 